\title{
Paramètres démographiques et économie de l'élevage camelin : une étude au Maroc
}

\author{
Lionel Julien ${ }^{1,2, *}$, Fatima Ezzahra Moutik ${ }^{3}$, Chaïmae Haloui ${ }^{3}$, Johann Huguenin ${ }^{1,2}$ \\ et Mohamed Taher Sraïri ${ }^{3}$ \\ ${ }^{1}$ CIRAD, UMR SELMET, F-34398 Montpellier, France \\ 2 SELMET, Univ Montpellier, CIRAD, INRA, Montpellier SupAgro, F-34398 Montpellier, France \\ ${ }^{3}$ Département des Productions et Biotechnologies Animales, Institut Agronomique et Vétérinaire Hassan II, B.P. 6202, Rabat, Maroc
}

\begin{abstract}
Résumé - Le dromadaire est capable de s'accommoder de l'hostilité du milieu désertique en mettant en valeur la végétation qui y est produite. Ces dernières années, il est l'objet d'un regain d'intérêt, eu égard à la qualité de ses produits et à ses caractéristiques physiologiques qui font de cette espèce l'une des plus aptes à s'adapter au changement climatique. Toutefois, les références sur ses performances zootechniques, et plus particulièrement sur les paramètres démographiques dans les systèmes naturels où il est exploité, demeurent limitées. Cette étude se propose d'analyser une partie des éléments démographiques en appliquant une méthode rétrospective de détermination de ces paramètres sur deux années successives, dans 122 troupeaux localisés dans la zone de M'Hamid El Ghizlane, au centre-est du Maroc. Les résultats ont démontré l'importante variabilité annuelle des paramètres démographiques, impactés par la disponibilité alimentaire des parcours, elle-même déterminée par la pluviométrie et sa répartition dans l'année. Les résultats ont aussi souligné la productivité limitée du dromadaire, ne dépassant pas en moyenne un chamelon viable pour trois chamelles en âge de reproduction. L'étude a révélé un potentiel de production de lait réduit dans un contexte de variabilité prononcée de l'offre alimentaire des parcours et d'éloignement des producteurs par rapport aux centres de consommation. En termes de pratiques d'alimentation, le recours à la complémentation est fréquent, sous forme de rebuts de dattes et d'orge subventionné. Les interventions sanitaires sont rares, du fait du pouvoir d'achat limité des éleveurs. Quant aux productions, elles sont surtout représentées par le croît du troupeau, les ventes de lait étant marginales. Quelques rares éleveurs recourent à la location de mâles pour des activités touristiques. Au final, les résultats confirment d'importantes marges d'amélioration de la productivité de l'élevage du dromadaire, qui demeure pour l'instant essentiellement au service de l'autoconsommation et de la capitalisation. Les marges dégagées ne permettent pas aux familles qui pratiquent cet élevage d'obtenir des revenus suffisants.
\end{abstract}

Mots clés : dromadaire / marges brutes / Maroc / paramètres démographiques / pratiques d'élevage

\begin{abstract}
Demographic parameters and profitability of camel breeding: a study in Morocco. The dromedary is able to adapt to the hostility of the desert environment by valorizing the vegetation produced there. In recent years, it attracted renewed interest, given the quality of its products and its physiological characteristics, which make this species one of the most appropriate for climate change adaptation. However, references on its breeding performances, and particularly on the demographic parameters in the natural systems where it is raised, remain limited. This study proposes to provide some demographic elements by using a retrospective method to determine these parameters over two successive years, in 122 herds located in the M'Hamid El Ghizlane area, in Central-Eastern Morocco. Results demonstrated an important annual variability of the demographic parameters, impacted by rangeland feed availability, which is determined by the annual level of rainfall and its monthly distribution. The results also emphasized the limited productivity of herds, as it did not exceed in average one camel calf per three mated she-camels. The results also showed limited milk yield given the variability in rangeland feed biomass and the long distances between production spots and consumption centers. With regard to feed supplementation, date wastes and subsidized barley grain are commonly used. Veterinary treatments are rare given the limited incomes of
\end{abstract}

\footnotetext{
$\overline{\text { *Auteur de correspondance }}$ : lionel.julien@cirad.fr
} 
breeders. The main production remains calf crop, as milk sales are limited. Very few breeders also rent adult males for touristic purposes. In summary, the results confirm significant margins of improvement in the productivity of camel breeding, particularly by a follow-up of reproduction practices, currently focused on self-consumption and capitalization. The margins generated do not allow families who breed camels to obtain sufficient incomes.

Keywords: demographic parameters / dromedary / Morocco / raw margins / rearing practices

\section{Introduction}

Avec un effectif de 35,5 millions d'individus et une contribution à l'approvisionnement mondial limitée $(0,2$ et $3,7 \%$ des viandes et du lait en 2018), les camelins sont considérés comme des espèces d'élevage d'intérêt secondaire (FAOSTAT, 2020). Ces dernières décennies, leurs prédispositions à la fourniture de produits de qualité nutritionnelle dans un contexte de dérèglement climatique (Zarrin et al., 2020) ont induit un regain d'intérêt pour leur élevage dans des territoires sous fortes contraintes. Longtemps utilisé comme animal de bât, indispensable au développement des échanges marchands entre les territoires oasiens (Faye et al., 2017), l'élevage du dromadaire subit les effets des récentes mutations socioéconomiques et environnementales. Dès lors, des stratégies d'adaptation ont été développées avec une orientation vers une marchandisation croissante des produits. Cet élevage assure, au-delà de ses fonctions premières de bât, orientées aujourd'hui vers le portage de touristes, une production de lait et/ou de viande, dans des zones éloignées des circuits de commercialisation des produits alimentaires. Malgré ses facultés adaptatives et la qualité nutritionnelle de ses produits, les références sur les paramètres démographiques du dromadaire dans les systèmes d'élevage où il est présent, demeurent rares en comparaison avec les autres espèces (Faye, 2018), l'essentiel étant issu d'essais en stations avec des effectifs limités. Or, dans les élevages extensifs, les performances reproductives sont des critères déterminants des productions de lait et de viande. L'usage de méthodes visant à la reconstitution des paramètres démographiques des troupeaux est donc d'une importance majeure (Kaufmann, 2005), ceux-ci synthétisant les caractéristiques biologiques des animaux et les modes de gestion mis en œuvre par les éleveurs (Tichit et al., 2004).

Dans les pays en développement, l'utilisation de méthodes appropriées pour évaluer ces paramètres constitue l'une des principales contraintes pour les élevages de dromadaires, du fait des difficultés de collecte de données fiables dans des troupeaux très mobiles et dispersés (Lesnoff et al., 2007). La mise en place de suivis individuels d'animaux peut être une solution, mais elle est très coûteuse (Bebe et al., 2002). Une autre voie serait la mise en place de suivis de troupeaux avec des procédures simplifiées (Madani et al., 2002), mais elle est compliquée dans un contexte de forte mobilité (nomadisme et transhumance). L'usage de méthodes alternatives, dites rétrospectives, basées sur une enquête unique annuelle par troupeau, peut être déployée dans un court laps de temps. En une seule visite, l'enquêteur reconstitue, après un recensement des animaux et avec le concours de l'éleveur, les événements démographiques survenus au cours de l'année (Lesnoff, 2013).

Parmi ces méthodes rétrospectives, la méthode «12 MO» basée sur un bilan de la reproduction et des flux des animaux (entrées et sorties) lors des 12 derniers mois, a été retenue (Lesnoff et al., 2008, 2013). Les données collectées et les méthodes d'analyses associées sont utiles pour évaluer les effets des variations interannuelles des taux démographiques. Malgré ses limites, dues en partie aux défauts de mémoire des éleveurs, ou à leurs réticences à restituer une information complète, elle constitue un outil intéressant et à coût limité pour une estimation des performances de l'élevage en conditions extensives. Elle est donc très adaptée pour l'espèce cameline, par définition caractérisée par une intense mobilité et une dispersion spatiale marquée. Dans le présent article, la méthode «12 $\mathrm{MO} »$ a été adoptée pour caractériser les performances de l'élevage de dromadaires dans une zone saharienne du Maroc. Les résultats et leurs conséquences sur les revenus des éleveurs seront discutés pour évaluer les perspectives d'évolution de cette activité.

\section{Matériel et méthodes}

L'étude a été réalisée dans la province de Zagora, au Centre Est du Maroc, plus exactement dans les communes de M'Hamid El Ghizlane $\left(2300 \mathrm{~km}^{2}\right)$ et de Tagounite $\left(4600 \mathrm{~km}^{2}\right)$ (Fig. 1). Elles sont situées dans l'étage bioclimatique saharien, avec une pluviométrie annuelle moyenne inférieure à $50 \mathrm{~mm}$ (mais variant amplement selon les années de 78 à $19 \mathrm{~mm} / \mathrm{an}$ entre 2010 et 2019) et des températures estivales de plus de $45^{\circ} \mathrm{C}$. L'agriculture oasienne pratiquée dans ces conditions souffre du stress hydrique et de la salinité de l'eau et des sols. L'élevage pastoral est donc primordial pour les revenus des ménages; il est constitué de troupeaux de camelins et caprins. Les effectifs camelins de la région ont varié entre 8720 et 12250 individus de 2008 à 2015, affectés par les flux d'animaux et les performances de reproduction. Les effectifs abattus contrôlés ne dépassaient pas 60 dromadaires par année (ORMVAO, 2018). La commercialisation des animaux hors de la région et le manque d'informations caractérisant la démographie des troupeaux sont autant de freins à l'évaluation de la productivité réelle des systèmes camelins.

Cette étude vise à cerner les performances démographiques des troupeaux camelins. La méthode de l'enquête rétrospective «12 MO» adoptée permet une estimation des paramètres démographiques annuels des troupeaux (Lesnoff, 2011). Elle consiste en un recensement du cheptel et des événements démographiques (naissances, décès naturels, abattages, prêts, achats, etc.) survenus dans les douze derniers mois. La méthode a été appliquée en 2018 et 2019, avec 62 puis 60 troupeaux étudiés. Ces derniers ont été choisis de manière à représenter la diversité des situations dans les deux communes. Ils ont été sélectionnés selon une méthode d'échantillonnage non probabiliste dite en réseau, où les éleveurs sont repérés à travers leurs connaissances. Les troupeaux choisis devaient 


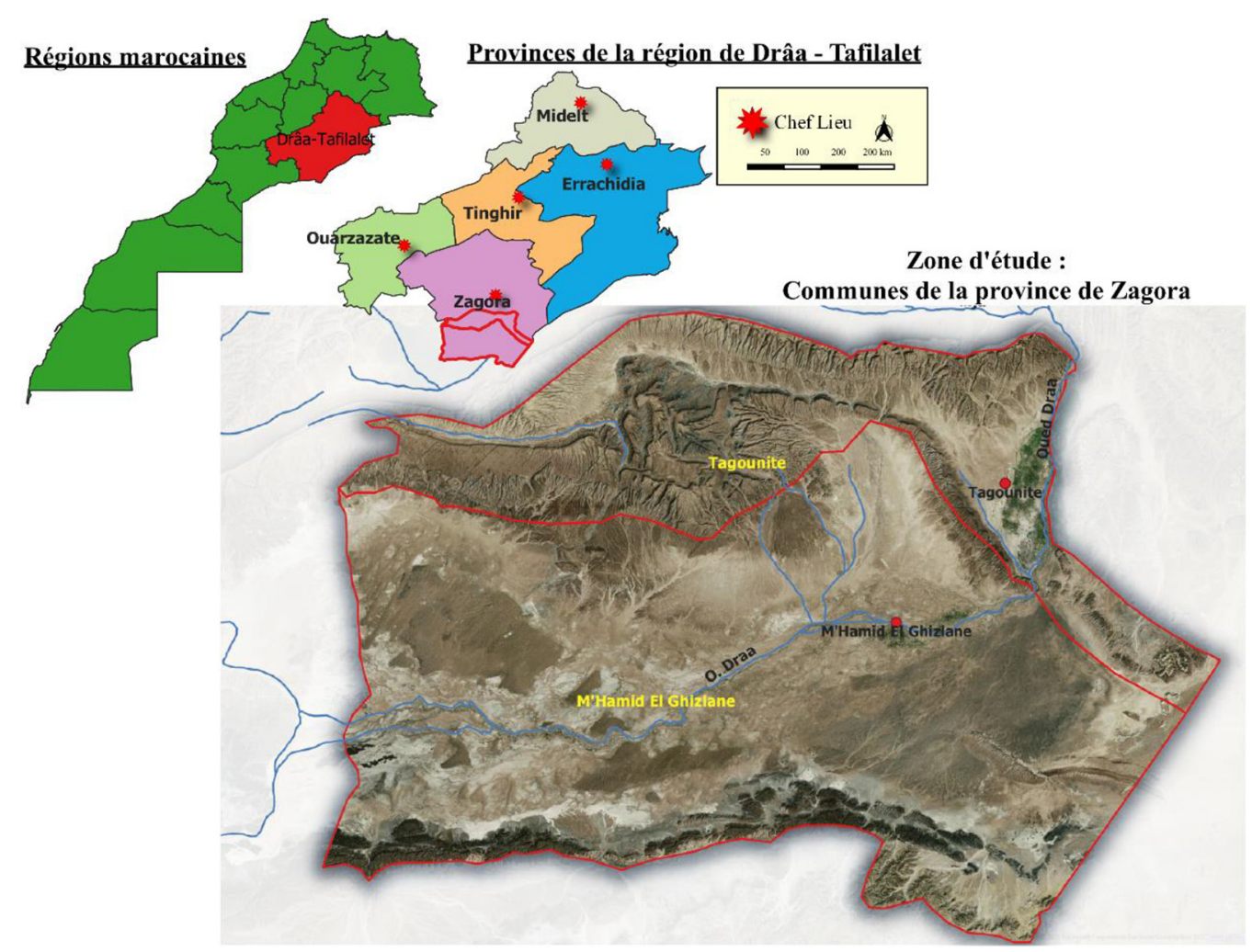

Fig. 1. Localisation de la zone d'étude.

Fig. 1. Location of the study area.

Tableau 1. Répartition des effectifs de dromadaires par classe d'âge et genre.

Table 1. Number of camels per age class and sex.

\begin{tabular}{lllllll}
\hline & F. JUV. & F. SAD. & F. ADU. & M. JUV. & M. SAD. & M. ADU. \\
\hline Effectifs & 398 & 798 & 2403 & 341 & 264 & 331 \\
Pourcentages & 8,8 & 17,6 & 53,0 & 7,5 & 5,8 & 7535 \\
\hline
\end{tabular}

F. : femelles ; M. : mâles ; 0 à 1 an : animaux non sevrés, nommés juvéniles (JUV.); 1 à 4 ans : nommés sub-adultes (SAD.), 4 ans étant considéré comme l'âge de la mise à la reproduction; Plus de 4 ans : animaux adultes (ADU.)

comporter impérativement des femelles reproductrices. Les enquêtes et observations d'animaux ont été réalisées soit aux abords des points d'eau, soit lors de sorties sur les parcours. Sur les 2 années, les éleveurs interrogés étaient issus de 4 tribus: les Aarib pour près de $70 \%$, les Ait Ounzar pour environ $25 \%$ et les Aït Sfoul et Aït Khbach pour seulement $5 \%$. Quelle que soit l'appartenance tribale, les éleveurs sont majoritairement originaires de la zone d'étude et illettrés à plus de $95 \%$. Ils sont relativement âgés, entre 22 et 82 ans, avec une moyenne de 52 ans et un âge médian de 50 ans. Cependant, ils ont tous une très forte expérience dans l'élevage camelin, avec en moyenne 40 ans et une présence dans le troupeau dès l'enfance (dès l'âge de 10 ans). L'ensemble des éleveurs interrogés ont une activité complémentaire à l'élevage de dromadaire. Près de $80 \%$ d'entre eux ont une activité agricole (phœniciculture et maraîchage) et $40 \%$ sont éleveurs de petits ruminants. Par ailleurs, environ $24 \%$ des éleveurs ont une activé salariée, soit dans le tourisme, soit comme fonctionnaire. En outre $16 \%$ des éleveurs interrogés sont des retraités, généralement de la fonction publique mais aussi du secteur agricole.

L'enquête a porté sur des effectifs totaux de 1929et 2060 animaux âgés de moins d'un an à 20 ans, en 2018 et 2019. Plus de $75 \%$ de ces effectifs étaient des femelles. La taille moyenne des troupeaux était de 37 individus, variant de 4 à 194. La répartition des individus par genre et classe d'âge est figurée dans le tableau 1 . Il en ressort une part prépondérante des chamelles adultes ( $53 \%$ des effectifs totaux) et des jeunes femelles conservées pour le renouvellement $(17,5 \%)$. Le nombre de géniteurs ( $>4$ ans) est limité à $7,2 \%$ car un reproducteur est indiqué pour 20 à 30 chamelles. Enfin, 16,3\% de l'effectif total sont des juvéniles : 8,8\% de femelles et 7,5\% de mâles non sevrés. 
Tableau 2. Taux de mise bas et d'avortement pour les deux années d'étude (en \%).

Table 2. Calving and abortion rates for the two study years (\%).

\begin{tabular}{|c|c|c|c|c|}
\hline & Classe d'âge & $\frac{2017}{\text { Moyenne } \pm \text { écart type }}$ & $\frac{2018}{\text { Moyenne } \pm \text { écart type }}$ & Test bilatéral ( $p$-value) \\
\hline Taux de mise bas & Adultes & $9,5 \pm 4,5$ & $8,5 \pm 2,5$ & 0,12 \\
\hline \multirow{2}{*}{ Taux d'avortement } & Adultes & $4,7 \pm 1,9$ & $3,0 \pm 0,9$ & 0,20 \\
\hline & Sub-adultes & $6,3 \pm 1,9$ & $5,9 \pm 2,4$ & 0,64 \\
\hline
\end{tabular}

Tableau 3. Taux de mortalité pour les deux années d'étude (en \%).

Table 3. Mortality rates for the tow study years (\%).

\begin{tabular}{|c|c|c|c|c|c|}
\hline & Genre & Classe d'âge & $\frac{2017}{\text { Moyenne } \pm \text { écart type }}$ & $\frac{2018}{\text { Moyenne } \pm \text { écart type }}$ & Test bilatéral ( $p$-value) \\
\hline & & Juvéniles & $11,9 \pm 9,4$ & $0 \pm 0$ & $1,1 \times 10^{-4}$ \\
\hline & $\mathrm{F}$ & Adultes & $1,1 \pm 0,9$ & $2,4 \pm 1,9$ & 0,27 \\
\hline & & Total & $4,4 \pm 3,5$ & $1,1 \pm 0,8$ & 0,12 \\
\hline & & Sub-adultes & $0,5 \pm 0,3$ & $1,2 \pm 1,2$ & 0,29 \\
\hline & $\mathrm{M}$ & Adultes & $2,7 \pm 2,2$ & $2,9 \pm 2,9$ & 0,31 \\
\hline & & Total & $6,4 \pm 5,6$ & $1,4 \pm 1,4$ & $2 \times 10^{-3}$ \\
\hline
\end{tabular}

Sur la base des données collectées, les performances des élevages ont été caractérisées par les principaux paramètres démographiques (taux de mise bas et de mortalité, avortement, etc.). Outre ces indicateurs, les caractéristiques des exploitations et les pratiques d'élevage ont été renseignées. Les données obtenues (complémentation alimentaire, pratiques sanitaires, usages de main-d'œuvre, valorisation et commercialisation du lait et du croît) ont permis d'appréhender la mise en marché des produits camelins et les revenus inhérents.

Une base de données a été conçue sur le logiciel ACCESS pour faciliter la saisie des informations collectées. Pour des raisons pratiques, une codification unique des questionnaires a été adoptée lors de la saisie. Un premier tri des variables a permis d'éliminer les données redondantes ou inintéressantes. Le recodage de variables continues en classes et la définition de modalités ont ensuite été effectués. La détermination des paramètres démographiques a été réalisée grâce au package «12 MO» (Lesnoff et al., 2013) développé par l'UMR SELMET (CIRAD) et adapté à l'enquête du même nom. Les distributions statistiques des différentes variables étudiées ne suivant pas une loi normale, les comparaisons des moyennes ont été réalisées à partir d'un test statistique non paramétrique (Wilcoxon-MannWhitney). La comparaison de ces critères sur les deux années d'études a permis d'une part d'estimer l'impact de l'aléa météorologique sur les troupeaux et d'autre part la réponse des éleveurs à cet aléa.

\section{Résultats}

L'analyse des paramètres de reproduction en fonction des classes d'âge montre une différence significative entre les deux années uniquement pour le taux de mise-bas des femelles adultes, le taux d'avortement ne suivant pas cette tendance (Tab. 2). Une chute de près de $25 \%$ du taux de mise bas des femelles adultes est ainsi enregistrée entre 2017 et 2018. L'étude a par ailleurs révélé une hausse significative des taux de mortalité des juvéniles mâles et femelles entre 2017 et 2018 , tendance qui ne se retrouve pas pour les autres catégories d'âge, mais qui impacte cependant le taux de mortalité totale dans le troupeau (Tab. 3).

Les analyses des taux d'exportation présentent une différence significative entre les deux années pour une seule catégorie d'animaux: les femelles sub-adultes ont connu en 2017 une plus forte exploitation qu'en 2018 (Fig. 2). Ce phénomène caractérise une forme d'adaptation des éleveurs au choc météorologique de 2017 (moindre pluviométrie et absence de précipitations au mois de mars) par un déstockage des animaux non productifs. Par ailleurs, il apparaît également un taux d'exploitation des mâles plus fort en 2017, quelle que soit leur classe d'âge, ce qui semble corroborer l'hypothèse d'un déstockage massif cette année-là pour répondre à la faible production de biomasse pastorale. Enfin, le taux d'importation, qui traduit la probabilité qu'un animal soit acheté, prêté ou confié durant une année, n'a pas connu de variation significative entre les deux années. 


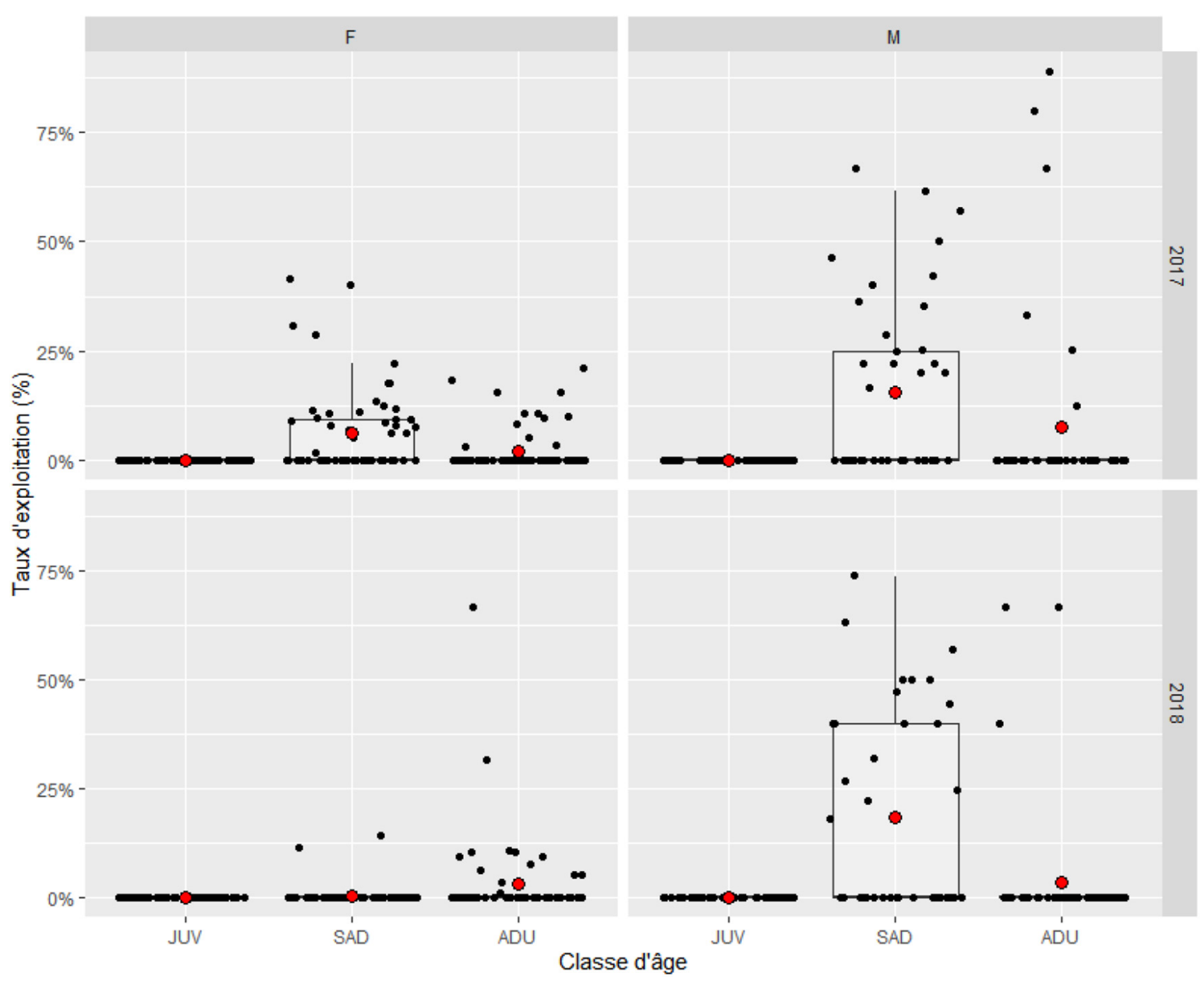

Fig. 2. Taux d'exploitation: le point rouge représente la médiane et les limites des boîtes le premier et le troisième quartile.

Fig. 2. Exploitation rate: The red dot represents median, borders of the boxes represent the first and third quartile.

En parallèle à l'étude des paramètres démographiques, l'évaluation des pratiques d'élevage du dromadaire a été effectuée. L'étude a révélé une mobilité lointaine limitée des troupeaux. La grande majorité $(79 \%)$ des élevages se caractérisent par la sédentarité ou des déplacements de moins de $50 \mathrm{~km}$. Seuls $21 \%$ des déplacements sont extrarégionaux, pour rejoindre les pâturages de Guelmim (à plus de $500 \mathrm{~km}$ au sud-ouest) en cas de précipitations favorables, avec un recours à des camions loués. Par ailleurs, la rareté des ressources pastorales spontanées, durant la très longue période estivale (d'avril à octobre), oblige les éleveurs à complémenter systématiquement les animaux. Les matières alimentaires utilisées sont des rebuts de dattes, de l'orge grain importée (subventionnée dans le cadre de programmes étatiques), voire de la luzerne locale (Fig. 3). Les quantités varient selon le stade physiologique de l'animal et son poids. En moyenne, $2,5 \mathrm{~kg}$ de grains d'orge et $4,0 \mathrm{~kg}$ de rebuts de dattes sont utilisés au quotidien par Unité Zootechnique cameline - UZc - lors de la période de complémentation (une chamelle et les différentes proportions des ateliers constituant sa suite). Les dépenses alimentaires annuelles correspondant à la complémentation, sont quasi similaires entre les deux années étudiées : 63 et 57 euros par UZc en 2017 et 2018 et les dépenses d'abreuvement (achats de citernes d'eau et leur acheminement) sont en moyenne de 5 euros par UZc et par an.

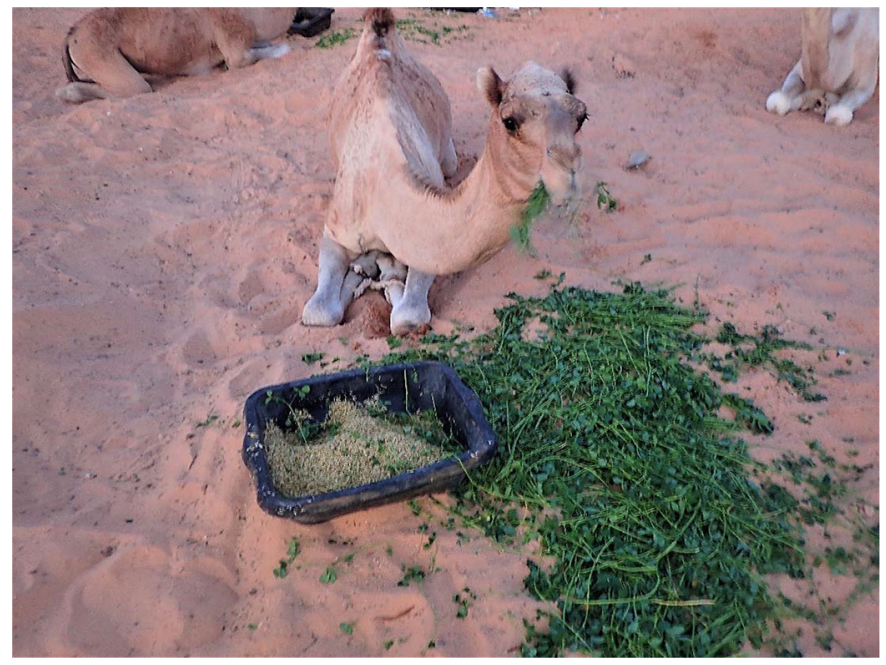

Fig. 3. Complémentation des dromadaires en orge et luzerne (CJulien).

Fig. 3. Camel supplementary feeding with barley and alfalfa (CJulien). 
Tableau 4. Bilan économique moyen de l'élevage camelin (euros/ Uzc et par an).

Table 4. Mean economic balance sheet of camel breeding (euros per Camel Unit-one she-camel and her siblings-per year).

\begin{tabular}{lcc}
\hline Année & 2017 & 2018 \\
\hline Revenus & & \\
$\quad$ Revenu viande & 114 & 89 \\
Revenu lait & 6 & 8 \\
Location de dromadaires pour le tourisme & 7 & 9 \\
Dépenses & & \\
Dépenses alimentaires et d'abreuvement & 68 & 62 \\
Dépenses vétérinaires & 0,2 & 0,2 \\
Dépenses de main-d'œuvre & 24 & 26 \\
Marge brute de l'activité cameline & 34,8 & 17,8 \\
\hline
\end{tabular}

La prévention et la lutte contre les principales pathologies (trypanosomose, variole, diarrhées, etc.) représentent un coût moyen annuel par élevage relativement limité : 18 et 15 euros/ UZc en 2017 et 2018. Le pouvoir d'achat plus que réduit des éleveurs et l'absence de vétérinaires spécialisés expliquent les faibles dépenses sanitaires. Les charges de main-d'œuvre sont aussi limitées, l'essentiel des personnes impliquées dans le gardiennage des troupeaux étant des membres de la famille, avec un rôle marqué des personnes âgées. Les dépenses de main-d'œuvre se trouvent dans les grands troupeaux (plus de 60 animaux), où elles atteignent en moyenne 24 et 26 euros/ UZc et par an, en 2017 et 2018. Les contraintes climatiques et le mode de vie entraînent un manque d'attractivité pour cette activité et les propriétaires de très grands troupeaux peinent à trouver et fidéliser une main-d'œuvre expérimentée, qui opte de plus en plus pour l'exode rural. Les revenus de cet élevage se sont réduits avec l'établissement de vastes zones militarisées aux frontières, entravant l'accès à une partie des ressources pastorales et points d'eau. Le bilan des recettes et dépenses annuelles de l'élevage camelin est consigné dans le tableau 4.

Les produits de l'élevage camelin sont le croît, le lait et éventuellement la location à des activités de portage. La productivité laitière est variable, de 0,25 à $5 \mathrm{l} / \mathrm{j}$ (après tétée du chamelon), avec une moyenne de 3 litres par jour et par chamelle. Une coopérative laitière a été créée en 2014 dans le village de M'Hamid El Ghizlane, mais les volumes de lait collectés restent limités avec une disponibilité aléatoire, impactée par les rythmes de mise bas et l'état des parcours (Fig. 4 et 5). Ces fluctuations sont principalement dues au mode de traite (après la tétée), à un long intervalle entre mise bas et à une valeur nutritive des parcours limitée. Ce marché est aussi contraint par un pouvoir d'achat local faible et des traditions culturelles des pasteurs nomades refusant en majorité la marchandisation du lait de chamelle. Sur les 62 éleveurs interrogés, seuls huit procèdent à des ventes épisodiques de lait, les quantités produites ne suffisant le plus souvent pas à satisfaire les besoins du chamelon. Le chiffre d'affaires du lait a été d'à peine 6 euros par UZc et par an en 2017 pour 8 euros en 2018. De ce fait, la vente des chamelons sevrés de plus d'un an, constitue l'essentiel des revenus des éleveurs. Par UZc, cela représente 114 et 89 euros en 2017 et

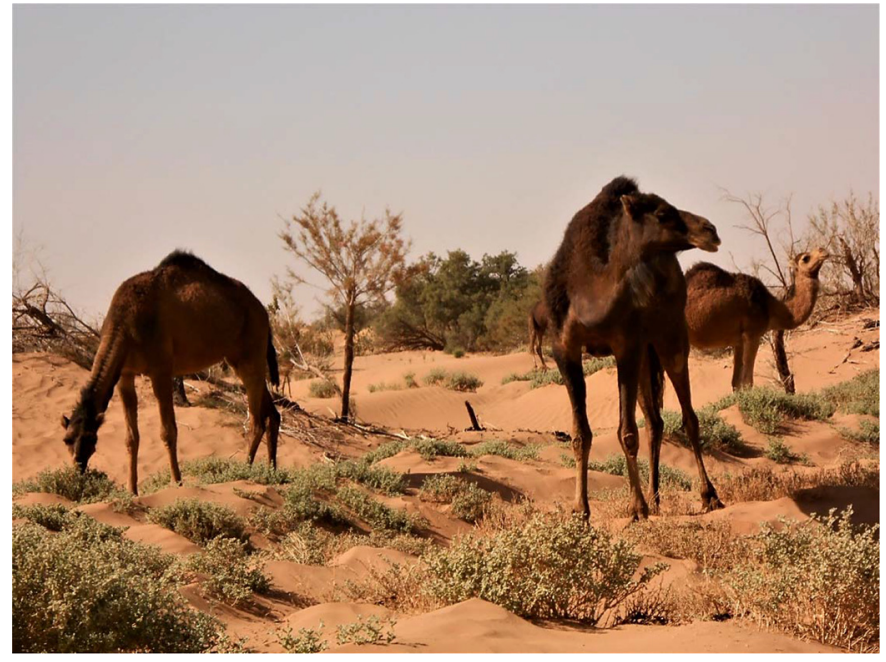

Fig. 4. Dromadaires en zone de parcours de printemps (@Sraïri).

Fig. 4. Dromedaries on spring pasture (@Srä̈ri).

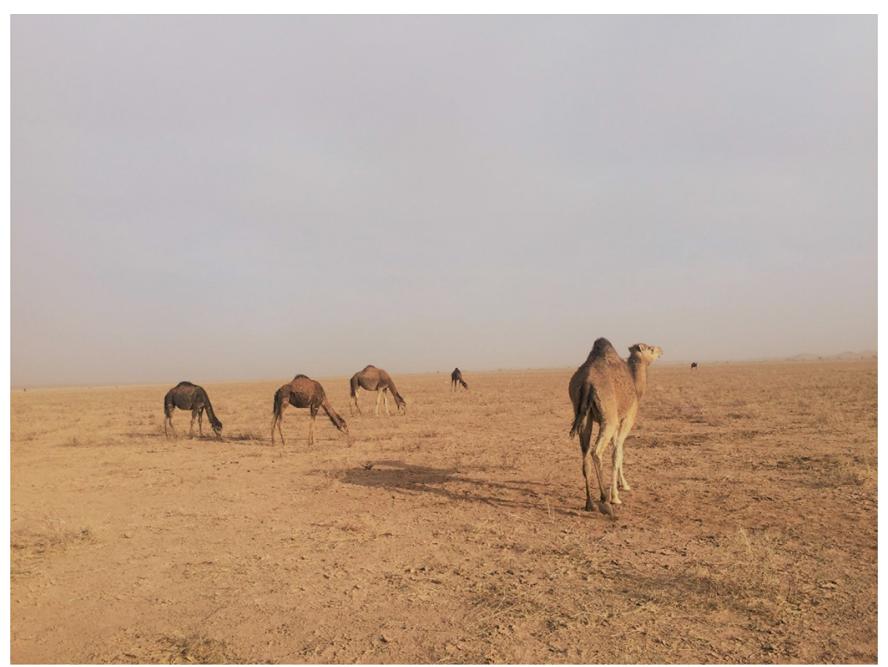

Fig. 5. Troupeau de dromadaires en zone de parcours proche de l'Erg Chegaga (@Julien).

Fig. 5. Herd of dromedaries in a rangeland near the Chegaga Erg (C)Julien).

2018. La différence s'explique surtout par les meilleures performances de reproduction enregistrées en 2017. Une dernière source de revenus originale de l'élevage camelin est la location des mâles pour des circuits touristiques. Cela a concerné 7 et 5 éleveurs sur 60 et 62, respectivement en 2017 et 2018. Les dromadaires sont loués via un réseau de connaissances, à la demande d'opérateurs touristiques qui recherchent des animaux dociles. Bien entendu, ces revenus demeurent très aléatoires, selon les flux touristiques. Ils ont représenté en moyenne 7 et 9 euros par UZc en 2017 et 2018.

Le bilan économique de l'élevage camelin illustre une baisse de près de $50 \%$ de la marge brute par UZc (de 34,8 à 17,8 euros) entre 2017 et 2018, correspondant presque 
exactement à la chute du taux de mise bas enregistrée entre ces deux années $(-45 \%)$. Il démontre que, à l'image de toute activité d'élevage naisseur, l'essentiel du résultat économique dépend de la capacité de reproduction des femelles, elle-même tributaire d'une alimentation suffisante et équilibrée et d'un état sanitaire convenable. Ces chiffres montrent des marges annuelles limitées: 440 et 481 euros par troupeau et par an. Bien entendu, ces moyennes voilent des disparités importantes, selon les effectifs d'UZc par troupeau, qui varient de 5 à 126. De manière générale, les revenus de l'élevage camelin sont insuffisants par rapport aux besoins des familles, surtout pour celles qui gèrent des troupeaux avec des effectifs limités (moins de 50 UZc). Cette analyse économique montre que l'élevage camelin au sens strict du terme n'est que très peu rentable, générant même des marges négatives dans les troupeaux ayant une mortalité des chamelons très élevée. Ces chiffres ne renseignent cependant pas les quantités autoconsommées de lait et même de viande (abattage non contrôlé sur les parcours), qui représentent une contribution dans l'approvisionnement lacté et carné des familles pratiquant cet élevage.

\section{Discussion}

Les résultats de cette étude confirment la productivité limitée de l'espèce cameline dans les écosystèmes naturels désertiques où elle évolue. En considérant la fluctuation annuelle des taux de mise bas et de mortalité, il apparaît qu'en moyenne moins d'un chamelon mâle ou femelle est produit pour trois femelles sub-adultes et adultes mises à la reproduction. Cela vient en majorité d'une durée de gestation longue (13 mois) et d'un intervalle moyen entre mises bas de près de 2 ans (Faye et al., 2014). En outre, l'étude montre que les éleveurs ont tendance à limiter la mise à la reproduction des femelles en cas de disette alimentaire. En effet, l'année 2017, qui correspond au taux de mise bas le plus élevé chez les femelles adultes (58,4\% par rapport à $32,6 \%$ en 2018$)$, est celle qui fait suite à une année plus arrosée ( $32 \mathrm{~mm}$ en 2016 par rapport à $19 \mathrm{~mm}$ en 2017) et avec des pluies automnales (octobre et novembre) et printanières (mars), tandis qu'en 2017 il n'y a eu aucune pluie au printemps. Toutefois, le meilleur taux de mise bas en 2017 a été déprécié par un taux de mortalité des juvéniles significativement plus élevé (respectivement 11,9 et 18,0\% pour les mâles et femelles, par rapport à 2018:0\% pour ces mêmes genres), expliqué par la disette engendrée par la moindre pluviométrie cette année-là. Ce taux de mortalité pour l'année 2017 reste proche de la valeur affichée par Saley (1990) pour des élevages au Niger (12 à $18 \%$ pour des chamelons de 6 à 12 mois). Les taux nuls de mortalité des chamelons en 2018 s'expliquent selon les déclarations des éleveurs par le nombre limité de mises bas qui a permis d'accorder plus de soins aux nouveau-nés. Cela s'est aussi conjugué à une moindre pluviométrie, qui a diminué la prolifération des insectes vecteurs de la trypanosomose. Cette dernière pathologie est réputée être particulièrement létale pour les jeunes chamelons là où les traitements vétérinaires sont peu accessibles et onéreux (Njiru et al., 2000). Nos résultats montrent aussi que l'idée répandue de la mobilité des troupeaux camelins, associée au nomadisme et à des déplacements de forte amplitude, est battue en brèche par les évolutions de la société et la montée en puissance des besoins des familles (aspirations à la scolarisation des enfants, recherche d'un mode de vie moins pénible avec l'électrification du domicile) qui ont plutôt induit la sédentarisation des éleveurs. De ce fait, les déplacements sont surtout de faible distance (moins de $50 \mathrm{~km}$ ) et seul un membre de la famille ou un ouvrier salarié est chargé de suivre les mouvements des animaux et de leur apporter les soins nécessaires (complémentation en aliments achetés, éventuels traitements vétérinaires, etc.). Ces tendances rejoignent les observations d'Alary et al. (2018) rapportées dans la même région d'étude et confirment les évolutions progressives des pratiques d'élevage du dromadaire.

En ce qui concerne la production de lait camelin, les résultats montrent que la vente de ce produit demeure peu répandue. Il faut y voir les effets cumulés de rendements moyens par chamelle généralement limités, suffisant à peine à répondre aux besoins du chamelon, et de la consommation de la famille de l'éleveur (autoconsommation) sous forme de lait et de petit lait (après fermentation). En cas d'excédents, et vu les distances importantes pour écouler le produit dans des circuits de commercialisation, peu d'éleveurs se résolvent à une vente régulière du produit. En outre, l'irrégularité de la production, issue des rythmes de mise bas et de la disponibilité alimentaire dans les parcours désertiques, décourage de nombreux éleveurs de pratiquer la vente systématique du lait, d'autant que le marché de M'Hamid El Ghizlane demeure de taille limitée; le prix du produit (près de 1,5 euro/litre) empêche la majorité de la population de l'acheter, à l'instar de ce que rapportent Kempen et al. (2016).

Il apparaît au final que l'élevage camelin, tout en étant bien adapté à un territoire soumis à des contraintes importantes, présente encore des marges importantes d'amélioration de sa productivité. Il doit ainsi être plus considéré sous l'angle de ses rôles culturels qu'économiques, puisqu'il s'accompagne d'un ensemble de rituels, le plus souvent collectifs (cérémonie du thé, gardiennage et mobilités collectives, etc.) qui participent du patrimoine immatériel, voire de l'identité, de ceux qui s'y adonnent. Une amélioration, même limitée, de la productivité de l'élevage camelin nécessiterait des interventions ciblées et une gouvernance optimale des chaînes de valorisation du croît dans les troupeaux et du lait. Cela pourrait assurer davantage de revenus aux populations locales, tout en leur procurant des produits de très haute qualité nutritionnelle. Cela pourrait aussi contribuer à limiter l'exode rural à partir des régions sahariennes, qui souffrent de déséquilibres démographiques pouvant entraver leur développement futur (Aït Hamza et al., 2010).

\section{Conclusion}

Cette étude visait à analyser l'efficacité économique et la durabilité des systèmes de production camelins dans des contextes spécifiques où l'élevage est un moyen d'adaptation au changement globaux (compétitivité internationale, changement social, dérèglement climatique). Elle met en évidence les paramètres démographiques limités et la faible productivité des élevages extensifs et semi-extensifs camelins de la région de M'Hamid El Ghizlane. La principale source de revenus de cet élevage demeure de loin les ventes de jeunes sevrés, au 
regard des volumes limités de lait commercialisés. Par ailleurs, les charges de production, principalement l'alimentation, ainsi que la main-d'œuvre, essentiellement pour les grands troupeaux, induisent de faibles revenus. L'importance des freins économiques et sociaux qui pèsent sur la filière cameline de la zone d'étude, associés à l'impact attendu du changement climatique, impliquent des perspectives d'évolution qui intiment un ensemble de précautions d'intervention et un renforcement de l'intégration des différents intervenants dans cette filière.

L'appui à cette filière nécessiterait d'être réalisé dans le cadre d'approches participatives impliquant l'ensemble des acteurs que sont les organisations professionnelles, les services locaux du développement agricole, et également le secteur de l'enseignement et de la recherche dans l'optique d'envisager des actions concrètes pour faire face à une demande croissante en produits camelins. L'un des enseignements majeurs de cette étude est la nécessité de cibler en premier lieu les paramètres de la reproduction et les mortalités. Les résultats montrent également des possibilités limitées d'intensification de la production de lait, avec cependant un potentiel de développement, mais qui va nécessiter une caractérisation plus fine des bassins de production ainsi que des termes de la demande (quantité, période, etc.). L'importance de l'exode rural à partir des régions sahariennes pèse sur le développement économique de ces territoires. $\mathrm{Y}$ encourager des systèmes de production aptes à mettre en valeur des ressources rares, comme l'élevage des camelins le permet, constitue indéniablement une opportunité à saisir. Outre les produits comestibles (viande et lait), il ne faut pas omettre les autres productions du dromadaire (cuir, poils, portage de touristes), ainsi que sa contribution aux services écosystémiques (entretien des espaces, patrimoine culturel, etc.), qui peuvent aussi augmenter les revenus des populations locales et contribuer à la renommée de la région.

Améliorer la rentabilité de cet élevage par une meilleure maîtrise de la reproduction et la baisse des mortalités, tout en diminuant sa pénibilité, s'avère impératif pour rendre cette activité plus attractive pour les jeunes générations.

Remerciements. Cette étude a été réalisée dans le cadre du projet CAMED (ERANET-MED2-72-367-CAMED) financé au Maroc par le ministère de l'Enseignement supérieur. L'appui de l'Office régional de mise en valeur agricole de Ouarzazate et de ses relais locaux à M'Hamid El Ghizlane pour l'accès aux troupeaux est dûment apprécié. La collaboration des éleveurs lors des entretiens a été déterminante. Ils en sont vivement remerciés.

\section{Références}

Aït Hamza M, El Faskaoui B, Fermin A. 2010. Les oasis du Drâa au Maroc. Hommes et migrations 1284: 56-69. DOI: 10.4000/ hommesmigrations. 1241.

Alary V, Sraïri MT, Amsidder L, Ramdane A, Benidir M, Julien L, et al. 2018. Main drivers of mobility changes within camel herds: between tradition and modernity. In: Présentation orale au $5^{e}$ congrès l'ISOCARD (International Society of Camelid Research and Development), Laâyoune, Maroc. http://www.iav.ac.ma/Iso card-Morocco2018/wp-content/uploads/2018/11/Brochure-Pro gram-A5-v-21-45.pdf.
Bebe BO, Udo HMJ, Thorpe W. 2002. Development of smallholder dairy systems in the Kenya highlands. Outlook on Agriculture 31: 113-120. DOI: 10.5367/000000002101293958.

FAOSTAT. 2020. Données sur les productions mondiales de lait et de viande pour les différentes espèces d'élevage. Rome: FAO. www. fao.org/faostat/fr/\#data [consulté le 8/4/2020].

Faye B. 2018. The improvement of camel reproduction performances: just a technical question? Revue Marocaine des Sciences Agronomiques et Vétérinaires 6: 265-269.

Faye B, Senoussi H, Jaouad M. 2017. Le dromadaire et l'oasis : du caravansérail à l'élevage périurbain. Cahiers Agricultures 26(1): 14001. DOI: 10.1051/cagri/2017005.

Faye B, Jaouad M, Bhrawi K, Senoussi A, Bengoumi M. 2014. Elevage camelin en Afrique du Nord : état des lieux et perspectives. Revue d'Elevage et de Médecine vétérinaire des Pays tropicaux 67 (4): 213-221. DOI: 10.19182/remvt.20563.

Kaufmann BA. 2005. Reproductive performance of camels (Camelus dromedarius) under pastoral management and its influence on herd development. Livestock Production Science 92(1): 17-29. DOI: 10.1016/j.livprodsci.2004.06.016.

Kempen E, Kasambala J, Christie L, Symington E, Jooste L, Van Eeden T. 2016. Expectancy-value theory contributes to understanding consumer attitudes towards cow's milk alternatives and variants. International Journal of Consumer Studies 41(3): 245252. DOI: $10.1111 /$ ijcs.12331.

Lesnoff M, Messad S, Juanès X. 2013. 12MO: A cross-sectional retrospective method for estimating livestock demographic parameters in tropical small-holder farming systems-Version 2. Montpellier, France: CIRAD (French Agricultural Research Centre for International Development). http://livtools.cirad.fr.

Lesnoff M. 2011. Démographie et zootechnie tropicales : un lien par les modèles matriciels appliqués aux cheptels de ruminants dans les élevages extensifs. Mémoire Habilitation à Diriger des Recherches, Université Montpellier II/Cirad, Montpellier, France.

Lesnoff M. 2013. Méthodes d'enquête pour l'estimation des taux démographiques des cheptels de ruminants domestiques tropicaux. Synthèse, limites et perspectives. Revue d'élevage et de Médecine vétérinaire des Pays tropicaux. 66 (2): 57-67. DOI: 10.19182/ remvt.10142.

Lesnoff M, Lancelot R, Moulin CH. 2007. Calcul des taux démographiques dans les cheptels domestiques tropicaux: approche en temps discret. Versailles, France: Editions Quae, 74 p.

Lesnoff M, Saley M, Adamou K, N'Djafa Ouaga H, Ayantunde A, Gérard B. 2008. 12MO: A retrospective method for estimating demographic parameters in tropical ruminant livestock populations. CIRAD (French Agricultural Research Centre for International Development), ILRI (International Livestock Research Institute.

Madani T, Hubert B, Vissac B, Casabianca F. 2002. Analyse de l'activité d'élevage bovin et transformation des systèmes de production en situation sylvopastorale algérienne. Revue d'Elevage et de Médecine vétérinaire des Pays tropicaux. 55(3): 197-209. DOI: $10.19182 /$ remvt.9824.

Njiru ZK, Ole-Mapeny IM, Ouma JO, Ndung'u JM, Olaho Mukani W. 2000. Prevalence of trypanosomosis in camel calves: A pilot study in Laikipia District of Kenya. Revue d'Elevage et de Médecine vétérinaire des Pays tropicaux 53(2): 183-186. DOI: 10.19182/remvt.9749.

ORMVA Ouarzazate (Office Régional de Mise en Valeur Agricole de Ouarzazate). 2018. Monographie de la zone d'action de l'ORMVAO. Ouarzazate, Maroc, 12 p.

Saley M. 1990. Performances de reproduction du dromadaire au Niger. In: Proceedings $d u$ Workshop "It is possible to improve 
reproductive performances in the camel?", 10-12 Sept 1990, Maisons-Alfort, IEMVT, Paris, France, pp. 379-386.

Tichit M, Ingrand S, Moulin CH, Cournut S, Lasseur J, Dedieu B. 2004. Analyser la diversité des trajectoires productives des femelles reproductrices : intérêts pour modéliser le fonctionnement du troupeau en élevage allaitant. INRA Productions
Animales 17 (2): 123-132. DOI: 10.20870/productions-anima les.2004.17.2.3560.

Zarrin M, Riveros JL, Ahmadpour A, de Almeida AM, Konuspayeva G, Vargas-Bello-Pérez E, et al. 2020. Camelids: new players in the international animal production context. Tropical Animal Health and Production (52): 903-913. DOI: 10.1007/s11250-019-02197-2.

Citation de l'article : Julien L, Moutik FE, Haloui C, Huguenin J, Sraïri MT. 2021. Paramètres démographiques et économie de l'élevage camelin: une étude au Maroc. Cah. Agric. 30: 1. 